\title{
Nanometer-scale studies of point defect distributions in GaMnAs alloys
}

\author{
J. N. Gleason, M. E. Hjelmstad, V. D. Dasika, ${ }^{\text {a) }}$ and R. S. Goldman ${ }^{\text {a),b) }}$ \\ Department of Materials Science \& Engineering, University of Michigan, Ann Arbor, Michigan 48109-2136 \\ S. Fathpour, S. Charkrabarti, and P. K. Bhattacharya \\ Department of Electrical Engineering and Computer Science, University of Michigan, Ann Arbor, Michigan \\ 48109-2122
}

(Received 30 June 2004; accepted 1 November 2004; published online 27 December 2004)

\begin{abstract}
We have investigated the concentrations and distributions of point defects in GaMnAs alloys grown by low-temperature molecular-beam epitaxy, using ultrahigh-vacuum cross-sectional scanning tunneling microscopy (XSTM). High-resolution constant-current XSTM reveals "A," "M," and "V" defects, associated with $\mathrm{As}_{\mathrm{Ga}}, \mathrm{Mn}_{\mathrm{Ga}}$, and $\mathrm{V}_{\mathrm{As}}$, respectively. $\mathrm{A}$ and $\mathrm{V}$ defects are present in all low-temperature-grown layers, while $\mathrm{M}$ defects are predominantly located within the GaMnAs alloy layers. In the GaMnAs layers, the concentration of $\mathrm{V}$ defects ([V]) increases with the concentration of $\mathrm{M}$ defects $([\mathrm{M}])$, consistent with a Fermi-level-dependent vacancy formation energy. Furthermore, $[\mathrm{M}]$ is typically two to three times $[\mathrm{A}]$ and $[\mathrm{V}]$, suggesting significant compensation of the free carriers associated with $\mathrm{Mn}_{\mathrm{Ga}}$. A quantitative defect pair correlation analysis reveals clustering of nearest $\mathrm{V}-\mathrm{V}$ pairs and anti-clustering of nearest $\mathrm{M}-\mathrm{M}, \mathrm{M}-\mathrm{V}$, and $\mathrm{M}-\mathrm{A}$ pairs. For all pair separations greater than $2 \mathrm{~nm}$, random distributions of defects are apparent. (C) 2005 American Institute of Physics. [DOI: 10.1063/1.1843284]
\end{abstract}

GaMnAs and related alloys are promising candidates for spintronic applications compatible with conventional GaAs technologies. The relative arrangements of $\mathrm{Mn}$ and other point defects in GaMnAs are expected to significantly impact its electronic and magnetic properties. ${ }^{1-3}$ At present, the nanometer-scale details of point defect distributions in GaMnAs are not well understood. A number of crosssectional scanning tunneling microscopy (XSTM) and scanning tunneling spectroscopy (STS) studies have reported the presence of various combinations of $\mathrm{Mn}_{\mathrm{Ga}}, \mathrm{As}_{\mathrm{Ga}}, \mathrm{V}_{\mathrm{As}}$, and $\mathrm{Mn}_{\mathrm{I}}$ in GaMnAs alloys. ${ }^{4-8}$ Although $\mathrm{Mn}_{\mathrm{Ga}}$ concentrations have been reported to increase with increasing $x$ in $\mathrm{Ga}_{1-x} \mathrm{Mn}_{x} \mathrm{As}$, ${ }^{5,8}$ conflicting results have been reported regarding the presence of $\mathrm{As}_{\mathrm{Ga}}$ and $\mathrm{V}_{\mathrm{As}}$, and the $\mathrm{Mn}$ composition dependence of their concentrations. ${ }^{4,5}$ In addition, a positron annihilation spectroscopy study revealed a decrease in $\mathrm{V}_{\mathrm{Ga}}$ concentration with increasing $\mathrm{Mn}$ composition in GaMnAs. ${ }^{9}$ The distributions of these defects and their dependence on Mn composition in GaMnAs remains unknown. Therefore, we have used XSTM to identify and quantify the concentration and distribution of "A," "V," and "M" defects associated with $\mathrm{As}_{\mathrm{Ga}}, \mathrm{V}_{\mathrm{As}}$, and $\mathrm{Mn}_{\mathrm{Ga}}$ in $\mathrm{Ga}_{1-x} \mathrm{Mn}_{x}$ As alloys with $0 \leqslant x \leqslant 0.05$. The concentration of $\mathrm{A}$ defects $([\mathrm{A}])$ is essentially independent of target $\mathrm{Mn}$ composition (for $x$ $>0.005$ ), while the concentrations of $\mathrm{V}$ and $\mathrm{M}$ defects ( [V] and $[\mathrm{M}]$ ) increase with target $\mathrm{Mn}$ composition. For all GaMnAs alloys, [M] is typically two to three times both [A] and $[\mathrm{V}]$, suggesting significant compensation of free carriers associated with $\mathrm{Mn}_{\mathrm{Ga}}$. A quantitative defect pair correlation analysis reveals clustering of nearest $\mathrm{V}-\mathrm{V}$ pairs, and anticlustering of nearest $\mathrm{M}-\mathrm{M}, \mathrm{M}-\mathrm{V}$, and $\mathrm{M}-\mathrm{A}$ pairs. Random distributions are observed for all defect pair separations $>2 \mathrm{~nm}$.

\footnotetext{
${ }^{\text {a) }}$ Also with: Department of Electrical Engineering and Computer Science.

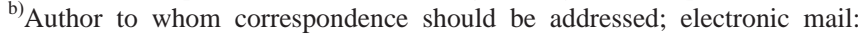
rsgold@umich.edu
}

The samples were grown on (001) $p^{+}$GaAs substrates, using molecular-beam epitaxy with an $\mathrm{As}_{4} / \mathrm{Ga}$ beamequivalent pressure ratio $\approx 15-20$. The heterostructures consisted of three sets of 10-period superlattices with alternating $\mathrm{Al}_{0.20} \mathrm{Ga}_{0.80} \mathrm{As}$ and $\mathrm{Ga}_{1-x} \mathrm{Mn}_{x}$ As layers with $x=0.005,0.025$, and 0.05. Each set of superlattices was separated by a multilayer consisting of 10- to 40-period AlAs/GaAs layers sandwiched between two $50 \mathrm{~nm}$ GaAs layers. The entire structure was grown at $250^{\circ} \mathrm{C}$ and capped with $300 \mathrm{~nm}$ GaAs. ${ }^{10}$ All Mn-free layers were Be-doped at $\sim 5$ $\times 10^{18} \mathrm{~cm}^{-3}$. For XSTM, the samples were cleaved to expose a (110) surface in an ultrahigh vacuum chamber with base pressure $<5 \times 10^{-11}$ Torr. Scanning tunneling microscopy was performed using commercially available $\mathrm{Pt} / \mathrm{Ir}$ tips. All images were obtained with a constant tunneling current of $0.15 \mathrm{nA}$ and sample bias voltages specified below.

Histograms of tip heights associated with bright or dark features (i.e., potential point defects) reveal two and three distinct modes for the GaAs and GaMnAs layers, respectively. The modes $0.3-0.9 \AA$ below, 1.2-3.0 $\AA$ above, and 0.5-1.4 $\AA$ above the GaAs background are attributed to point defects termed $\mathrm{V}, \mathrm{A}$, and $\mathrm{M}$, associated with $\mathrm{V}_{\mathrm{As}}, \mathrm{As}_{\mathrm{Ga}}$, and $\mathrm{Mn}_{\mathrm{Ga}}$. To further distinguish $\mathrm{A}$ and $\mathrm{M}$ defects, the lateral positions and characteristic features of each defect were also considered, as shown in the high resolution XSTM image of a $\mathrm{Ga}_{0.95} \mathrm{Mn}_{0.05}$ As layer in Fig. 1(a). Figures 1(b)-1(d) present cuts of the tip height defined by the dashed, dotted, and solid lines in Fig. 1(a), for A, M, and V defects, respectively. As shown in Fig. 1(b), the A defects consist of hillocks spanning two [001] rows, offset by $1.9 \pm 1.0 \AA$ from the [001] row. The lateral extents of the A defects are similar to earlier XSTM studies of $\mathrm{As}_{\mathrm{Ga}}$ in GaAs; ;,11 a lack of visible satellites for on-surface $\mathrm{As}_{\mathrm{Ga}}$ has also been reported in $\mathrm{GaAs}^{12}$ and GaMnAs. ${ }^{8}$ The $\mathrm{M}$ defects, shown in Fig. 1(c), consist of hillocks with a shoulder, which are offset from the [001] row by $0.7 \pm 0.4 \AA$, similar to earlier XSTM reports of $\mathrm{Mn}_{\mathrm{Ga}} \cdot{ }^{5,8} \mathrm{In}$ order to quantify the concentration and distribution of point defects in GaAs and GaMnAs, we applied these criteria to 


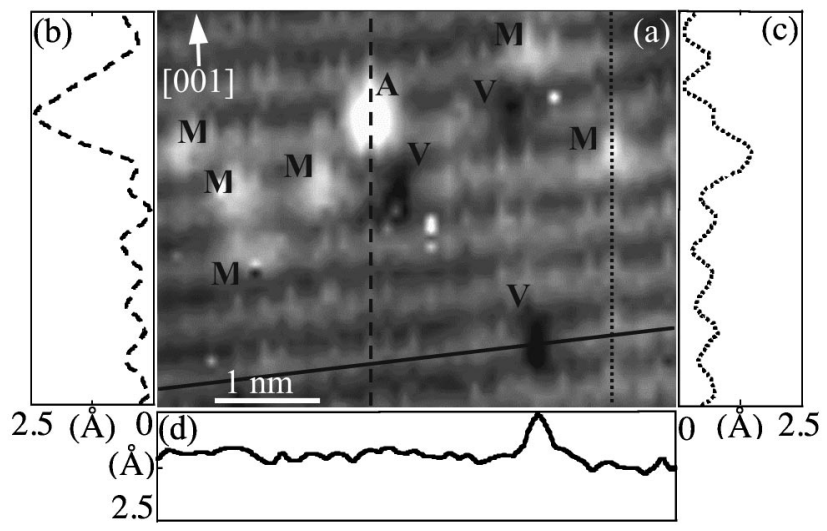

FIG. 1. (a) High resolution XSTM topographic image of $\mathrm{Ga}_{0.95} \mathrm{Mn}_{0.05} \mathrm{As}$, acquired at a sample bias voltage of $-2.0 \mathrm{~V}$. The gray-scale range displayed is $1.9 \AA$. Cuts of the tip height along the dashed, dotted, and solid lines in (a) are displayed in (b), (c), and (d) for A, M, and V defects, respectively.

several high resolution XSTM images spanning a total area greater than $0.2 \mu \mathrm{m}^{2}$.

Figure 2(b) presents a representative high resolution XSTM image containing three periods of $\mathrm{Ga}_{0.95} \mathrm{Mn}_{0.05} \mathrm{As} /$ $\mathrm{Al}_{0.20} \mathrm{Ga}_{0.80}$ As with $\mathrm{A}, \mathrm{V}$, and $\mathrm{M}$ defects identified with red ovals, green squares, and blue circles, respectively. To identify the interfaces, a cut of the tip height was averaged across the step free region of the image, shown as the blue line in Fig. 2(a). To remove the [001] atomic periodicity, frequencies less than $20 \AA$ were filtered out, as illustrated by the orange line in Fig. 2(a). Finally, the inflection points in the orange line in Fig. 2(a) were defined as the interface positions, denoted as red dashed lines in Fig. 2(b). Figure 2(b) suggests that $\mathrm{M}$ defects are typically restricted to GaMnAs layers, while $\mathrm{A}$ and $\mathrm{V}$ defects appear throughout the image. In the GaAs layers, $[\mathrm{M}] \sim 10^{19} \mathrm{~cm}^{-3}$ may be due in part to misidentified $\mathrm{Be}_{\mathrm{Ga}}{ }^{12}$ As will be shown in Fig. $3,[\mathrm{M}]$ in the $\mathrm{GaAs}$ layers is significantly lower than the error bars for $[\mathrm{M}]$ in the GaMnAs layers. All the defects identified were assumed to be located in the topmost plane of the cleaved

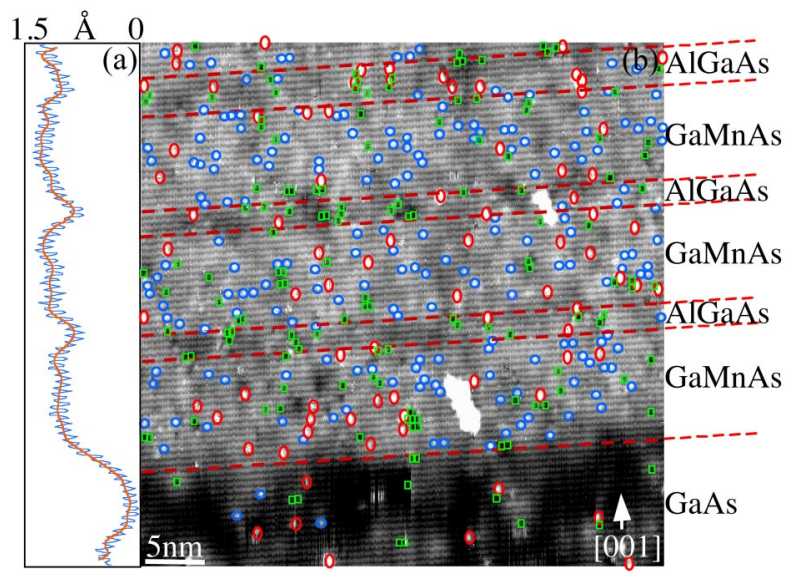

FIG. 2. (Color online) (b) High resolution XSTM topographic image of $\mathrm{Ga}_{0.95} \mathrm{Mn}_{0.05} \mathrm{As}_{\mathrm{Al}} / \mathrm{Al}_{0.3} \mathrm{Ga}_{0.7} \mathrm{As}$ superlattices, obtained at a sample bias voltage of $-2.0 \mathrm{~V}$. The gray scale range displayed is $2.6 \AA$. A, M, and V defects are highlighted with red ovals, blue circles, and green squares, respectively. A cut of the tip height averaged over $\sim 1 / 2$ the image is shown as the blue line in (a). The orange line in (a) represents the averaged tip height with frequencies less than $20 \AA$ removed. Inflection points in the orange line are defined as the interface positions, labeled as red dashed lines in (b).

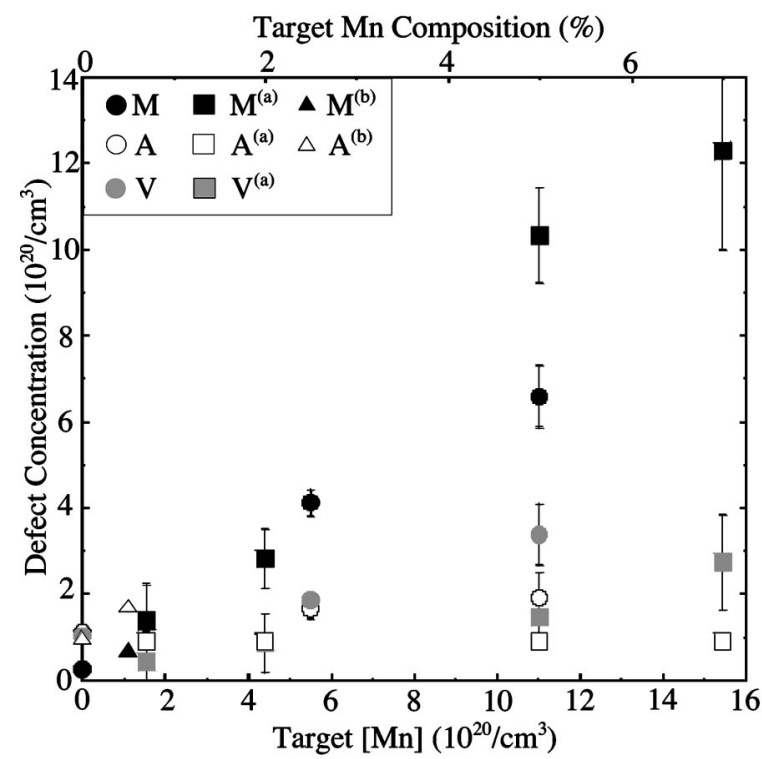

FIG. 3. Concentrations of M, V, and A defects as a function of target $\mathrm{Mn}$ concentration for $\mathrm{Ga}_{1-x} \mathrm{Mn}_{x}$ As. (a) See Ref. 8. (b) See Ref. 5.

surface, resulting in an effective defect "depth" of one-fourth the unit cell face diagonal $(\sim 2.00 \AA) .{ }^{13}$

Figure 3 shows a plot of $[\mathrm{A}],[\mathrm{V}]$, and $[\mathrm{M}]$ versus target $\mathrm{Mn}$ composition, for a variety of layers. In our GaAs and GaMnAs layers, $[\mathrm{A}]$ is $\sim 1.0$ and $\sim 1.7 \times 10^{20} \mathrm{~cm}^{-3}$, respectively, similar to earlier reports. ${ }^{5,8,13,14}$ In general, [V] increases with target Mn composition, supporting earlier predictions of decreasing $\mathrm{V}_{\mathrm{As}}$ formation energy as the Fermi level moves toward the GaAs valence band edge. ${ }^{15,16}$ Interestingly, our layers contain about twice the $[\mathrm{V}]$ as those of Mikkelsen et al., ${ }^{8}$ presumably due to the increased As incorporation and consequent lower $[\mathrm{V}]$ for growth using an $\mathrm{As}_{2}$ source. ${ }^{17}$ Finally, $[\mathrm{M}]$ increases with target $\mathrm{Mn}$ composition, similar to earlier reports. ${ }^{5,8}$ However, in our case, [M] $\approx 2 / 3[\mathrm{Mn}]$. The discrepancy between $[\mathrm{M}]$ and $[\mathrm{Mn}]$ may be due in part to a significant fraction of interstitial $\mathrm{Mn}\left(\mathrm{Mn}_{\mathrm{I}}\right)$ in GaMnAs. Rutherford backscattering spectrometry and particle-induced $\mathrm{x}$-ray emission measurements have suggested up to $7 \% \mathrm{Mn}_{\mathrm{I}} ;{ }^{18}$ yet, $\mathrm{Mn}_{\mathrm{I}}$ have not been observed in filled state STM images, presumably due to Coulombic repulsion between the STM tip and $\mathrm{Mn}_{\mathrm{I}}{ }^{4}$. Furthermore, $\mathrm{Mn}_{\mathrm{I}}-\mathrm{Mn}_{\mathrm{Ga}}$ complexes, similar to those predicted and observed by Sullivan et al., ${ }^{4}$ have not been observed, possibly due to probe tip resolution limitations. For target Mn compositions of $x=0.025$ and 0.05 , the ratio of $[\mathrm{A}]$ to $[\mathrm{M}]$ is $\sim 1 / 3$, while the ratio of $[\mathrm{V}]$ to $[\mathrm{M}]$ is $\sim 1 / 2$. Assuming all defects observed by XSTM are electrically active, A and V defects would each produce two to three free electrons, ${ }^{16,19}$ while $\mathrm{M}$ defects would produce one free hole. We note that in the GaAs layers, with a doping concentration of $\sim 5$ $\times 10^{18} \mathrm{~cm}^{-3}$, band-bending surrounding the point defects is evident. The apparent lack of band-bending surrounding A, $\mathrm{V}$, and $\mathrm{M}$ defects in GaMnAs is likely due to the high free carrier concentrations in these layers, similar to earlier work. ${ }^{4,5,8}$ Therefore, given the observed $[\mathrm{A}]$ to $[\mathrm{M}]$ and $[\mathrm{V}]$ to $[\mathrm{M}]$ ratios, these defects may contribute to the significant compensation of the free holes associated with $\mathrm{Mn}_{\mathrm{Ga}}$, as has been suggested by earlier STS studies. ${ }^{6}$

To determine the distributions of point defects in GaMnAs, we identified the locations of and separations be- 


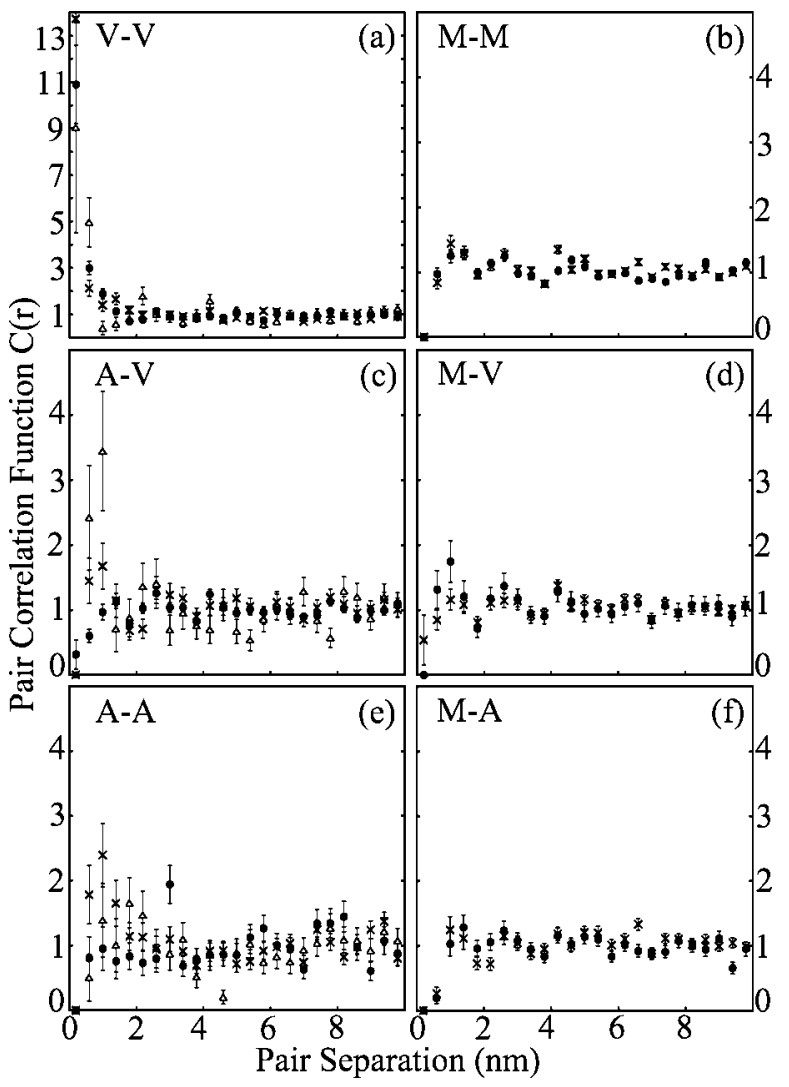

FIG. 4. Pair correlation functions, $C(r)$, vs pair separation for (a) V-V, (b) $\mathrm{M}-\mathrm{M}$, (c) $\mathrm{A}-\mathrm{V}$, (d) $\mathrm{M}-\mathrm{V}$, (e) $\mathrm{A}-\mathrm{A}$, and (f) $\mathrm{M}-\mathrm{A}$ pairs in GaAs (open triangles), $\mathrm{Ga}_{0.925} \mathrm{Mn}_{0.025} \mathrm{As}$ (crosses), and $\mathrm{Ga}_{0.95} \mathrm{Mn}_{0.05} \mathrm{As}$ (black circles).

tween $\mathrm{A}, \mathrm{V}$, and $\mathrm{M}$ defects in the GaMnAs layers. Defect pair separations were binned at $4 \AA$ intervals, yielding the number of experimental pairs, $N_{\text {expt. }}$, in shells of multiples of $4 \AA$. For the random distributions, we used the experimentally determined concentrations of point defects to determine the fraction of sites occupied by A, M, and V defects, and then distributed these randomly. Using these computergenerated distributions of $\mathrm{A}, \mathrm{V}$, and $\mathrm{M}$ defects, we identified the locations and separations between all defect pairs. The random defect pair separations were binned at $4 \AA$ intervals, yielding the number of random pairs, $N_{\text {rand }}$, observed within each shell in multiples of $4 \AA$. We generated more than 100000 random distributions and averaged the resulting number of random defect pairs. Finally, the pair correlation function, $C(r)$, consisted of $N_{\text {expt. }} / N_{\text {rand }}$, as a function of pair separation, with error bars determined by counting statistics. ${ }^{20}$ Using this treatment, positive and negative deviations of $C(r)$ from unity indicate a pair concentration higher or lower than a random distribution.

The pair correlation functions, $C(r)$, versus pair separation are shown in Fig. 4 for (a) V-V, (b) M-M, (c) A-V, (d) $\mathrm{M}-\mathrm{V}$, (e) A-A, and (f) $\mathrm{M}-\mathrm{A}$ pairs in $\mathrm{Ga}_{1-x} \mathrm{Mn}_{x}$ As. $C(r)$ associated with $x=0,0.025$ and 0.05 are shown as open triangles, crosses, and black circles, respectively. For all pair separations greater than $2 \mathrm{~nm}, C(r) \approx 1$, suggesting random distributions. In addition, $C(r)$ exhibits an oscillation with a period of $\sim 4-5$ lattice sites. This oscillation might be related to the periodicity of the surface reconstruction during growth, similar to earlier reports of InGaAs alloys. ${ }^{21,22}$

For pair separations less than $2 \mathrm{~nm}$, we first consider those defects present in all the $\mathrm{Ga}_{1-x} \mathrm{Mn}_{x}$ As layers. Figure 4(a) reveals a $C(r)$ greater than unity for the first and second nearest $\mathrm{V}-\mathrm{V}$ pairs, suggesting that vacancy clustering is significant in all layers, independent of Mn composition. In the case of A-V and A-A pairs shown in Figs. 4(c) and 4(e), $C(r)$ values less than unity suggest anti-clustering of the nearest $\mathrm{A}-\mathrm{V}$ and $\mathrm{A}-\mathrm{A}$ pairs. However, the $\sim 5 \AA$ lateral extent typical of A defects may mask the presence of nearest A-A pairs, resulting in an artificially low value of $C(r)$ in that case. In addition, Mn-composition dependent clustering of the second and third nearest A-V pairs is apparent in Fig. 4(c), possibly related to the Mn-composition dependent concentration of $\mathrm{V}$ defects discussed earlier.

For the defects in Mn-containing layers, random distributions are evident for all pair separations greater than $1 \mathrm{~nm}$. Figure 4(b) reveals $C(r)$ values less than unity, suggesting anti-clustering of the nearest $\mathrm{M}-\mathrm{M}$ pairs. Similar anticlustering has been observed for nearest In pairs in dilute InGaAs alloys, ${ }^{21}$ and for nearest $\mathrm{Zn}$ pairs in heavily Zn-doped GaAs. ${ }^{23}$ In those cases, In-In and $\mathrm{Zn}-\mathrm{Zn}$ pair repulsion was attributed to short-range strain ${ }^{21}$ and Coulombic repulsion ${ }^{23}$ effects, both of which may contribute in our case. For M-V and M-A pairs shown in Figs. 4(d) and 4(f), $C(r)$ values less than unity suggest anti-clustering of the nearest $\mathrm{M}-\mathrm{V}$ and $\mathrm{M}-\mathrm{A}$ pairs. It is interesting to note that $\mathrm{M}-\mathrm{V}$ anti-clustering is dependent on Mn composition, suggesting a pair anti-correlation energy which decreases with increasing Mn composition.

This work was supported by the Office of Naval Research under Grant No. N000-021-0899.

${ }^{1}$ R. N. Bhatt, M. Berciu, M. P. Kennett, and X. Wan, J. Supercond. 15, 71 (2002).

${ }^{2}$ C. Timm, J. Phys.: Condens. Matter 15, 1865 (2003).

${ }^{3}$ S. Sanvito and N. A. Hill, J. Magn. Magn. Mater. 242, 441 (2002).

${ }^{4}$ J. M. Sullivan, G. I. Boishin, L. J. Whitman, A. T. Hanbicki, B. T. Jonker, and S. C. Erwin, Phys. Rev. B 68, 235324 (2003).

${ }^{5}$ B. Grandidier, J. P. Nys, C. Delerue, D. Stievenard, Y. Higo, and M. Tanaka, Appl. Phys. Lett. 77, 4001 (2000).

${ }^{6}$ G. Mahieu, P. Condette, B. Grandidier, J. P. Nys, G. Allan, D. Stievenard, P. Ebert, H. Shimizu, and M. Tanaka, Appl. Phys. Lett. 82, 712 (2003).

${ }^{7}$ T. Tsuruoka, R. Tanimoto, N. Tachikawa, S. Ushioda, F. Matsukura, and H. Ohno, Solid State Commun. 121, 79 (2002).

${ }^{8}$ A. Mikkelsen, B. Sanyal, J. Sadowski, L. Ouattara, J. Kanski, S. Mirbt, O. Eriksson, and E. Lundgren, Phys. Rev. B 70, 085411 (2004).

${ }^{9}$ F. Tuomisto, J. Slotte, K. Saarinen, and J. Sadowski, Acta Phys. Pol. A 103, 601 (2003).

${ }^{10}$ T. Hayashi, M. Tanaka, T. Nishinaga, H. Shimada, H. Tsuchiya, and Y. Otuka, J. Cryst. Growth 175, 1063 (1997).

${ }^{11}$ K. Maeda, A. Hida, Y. Iguchi, Y. Mera, and T. Fujiwara, Mater. Sci. Semicond. Process. 6, 253 (2003).

${ }^{12}$ P. Ebert, Curr. Opin. Solid State Mater. Sci. 5, 211 (2001).

${ }^{13}$ D. C. Look, Thin Solid Films 231, 61 (1993).

${ }^{14}$ R. M. Feenstra, J. M. Woodall, and G. D. Pettit, Phys. Rev. Lett. 71, 1176 (1993).

${ }^{15}$ S. B. Zhang and A. Zunger, Phys. Rev. Lett. 77, 119 (1996).

${ }^{16}$ K. M. Luken and R. A. Morrow, Semicond. Sci. Technol. 11, 1156 (1996).

${ }^{17}$ M. R. Melloch, K. Mahalingam, N. Otsuka, J. M. Woodall, and A. C. Warren, Philos. Mag. A 67, 1495 (1993).

${ }^{18}$ K. M. Yu, W. Walukiewicz, T. Wojtowicz, I. Kuryliszyn, X. Liu, Y. Sasaki, and J. K. Furdyna, Phys. Rev. B 65, 201303 (2002).

${ }^{19}$ J. E. Northrup and S. B. Zhang, Phys. Rev. B 50, 4962 (1994).

${ }^{20}$ H. A. McKay, R. M. Feenstra, T. Schmidtling, and U. W. Pohl, Appl. Phys. Lett. 78, 82 (2001).

${ }^{21}$ K.-J. Chao, C. K. Shih, D. W. Gotthold, and B. G. Streetman, Phys. Rev. Lett. 79, 4822 (1997)

${ }^{22}$ B. Shin, B. Lita, R. S. Goldman, J. D. Phillips, and P. K. Bhattacharya, Appl. Phys. Lett. 81, 1423 (2002).

${ }^{23}$ P. Ebert, T. J. Zhang, F. Kluge, M. Simon, Z. Y. Zhang, and K. Urban, Phys. Rev. Lett. 83, 757 (1999). 